\title{
The Atlas Tile Calorimeter experience with 10,000 readout Photomultipliers operating since the start of the $p$ - $p$ collisions at LHC
}

\author{
Hadar Lazar, on behalf of the ATLAS Tile Calorimeter System \\ University of Chicago, United States
}

\begin{abstract}
The channels of TileCal, the hadron calorimeter of the ATLAS experiment at the LHC, is readout with 8-stage fine-mesh photomultipliers (PMTs), a special version of the Hamamatsu model R5900. About 10,000 PMTs are operating in TileCal. The PMT response stability allows to calibrate accurately the calorimeter and to achieve high performance of the energy reconstruction of the cells. Currently, no PMT replacement is foreseen before completion of the High Luminosity program of the LHC collider in the next decade. In this perspective, a number of measurements and tests are in progress to qualify the PMT robustness in terms of lifetime and response stability. Data from the Tile calibration procedure for the detector PMTs and from laboratory tests of spare PMTs are being analysed. Results on PMT failures, gain loss and quantum efficiency loss are presented. Analysis is focused on the study of the observed down-drift with time of the PMT response as a function of the integrated anode charge, and depending on the individual cell exposure to irradiation during the LHC operation. The multistage calibration system of Tile and the algorithms adopted to disentangle between gain and quantum efficiency loss are described, as well as the tests performed in dedicated test-benches.
\end{abstract}

Keywords: ATLAS, Tile Calorimeter, Laser II System, PMT response

\section{Introduction}

The Tile Calorimeter (TileCal) is a hadronic calorimeter in the central region of the ATLAS detector in CERN's Large Hadron Collider (LHC). 
Plastic scintillator tiles sample the energy deposited in the detector. Light produced by these tiles is transmitted by optical fibers from each cell to two photomultipliers (PMTs) located at the outer radius of the TileCal detector. TileCal is divided into four partitions of 64 modules in azimuth, with each module in the long barrel containing 45 PMTs and each module in the two extended barrels containing 32 . This totals to about 10,000 8-stage fine-mesh PMTs, a special version of the Hamamatsu model R5900, that read the light output of all scintillator tiles.

The TileCal calibration system [1] has been used to monitor the performance of its readout electronics since Run 1, which spanned from 2009-12, as well as during the ongoing Run 2, which started in 2015 [2]. This has involved monitoring the response stability of the 10,000 PMTs, a key function that is necessary for an accurate calibration of signals and reconstruction of events. This article presents PMT response studies conducted using the Laser calibration system to determine response down-drift and thus anticipate future PMT performance in the ATLAS detector. Furthermore, it discusses a test bench setup in the Pisa-INFN labs that studied the relationship between PMT response down-drift and absolute gain loss, which ultimately determined a new statistical method to more accurately calculate PMT absolute gain.

\section{Setup of the Laser calibration system}

\subsection{The ATLAS Detector}

The three main calibration systems of TileCal - the Cesium system, Laser system, and Charge Injection System (CIS) - are used to calculate energy scales and constants to convert channel readouts to energy units of $\mathrm{GeV}$, as well as determine hardware performance and identify hardware malfunctions. As illustrated in Figure 1 [3], each system tests a specific element of the readout chain. The Cesium system tests the stability and relative optical response of every scintillator tile, the Laser system analyzes the stability of PMTs, and finally the CIS tests the readout electronics, including the Analog to Digital Converters.

In dedicated Laser runs taken between LHC fills several times a week, the Laser calibration system sends laser pulses of known intensities into the PMTs' photocathodes and collects data in low gain. Laser calibration factors calculated from this data are used to monitor and calibrate the gain linearity of each PMT. In late 2014, the Laser I system was replaced with a Laser II 


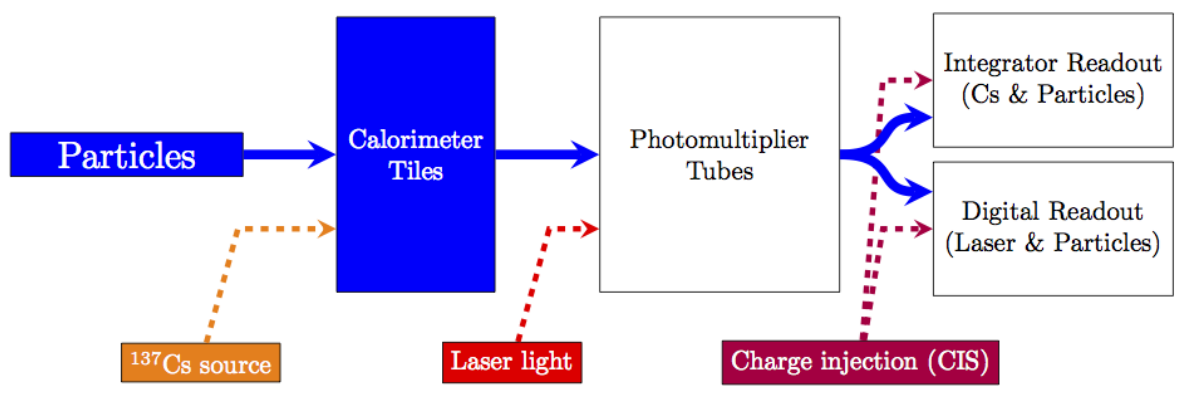

Figure 1: Calibration of readout chain

system, pictured in Figure 2 [4,5]. The Laser II system aims to reduce the contribution of the calibration uncertainty to below the level of the irreducible systematics in the determination of the Jet Energy Resolution and Jet Energy Scale. Figure 3 summarizes the stability of the Laser II system, which was determined by responses collected over the course of three months for diodes that were placed at different readout steps of the system. Groups of three or four diodes were used to monitor the laser intensity, light transmitted after the filter wheel, and light at the exit of the final beam expander.

\subsection{Laser Test Bench}

Nine PMTs that were dismounted from the ATLAS detector in February 2017 were tested using a test bench setup located at the Pisa-INFN labs. These PMTs read signals from different cell types and collected data from Run 1 and Run 2. At the test bench, measurements of PMT absolute gain and the absolute and normalized response were taken using stable laser pulses of $437 \mathrm{~nm}$.

\section{Response measurements}

Within the ATLAS detector, the Laser calibration system found the response variation relative to the first day of observation of each PMT. For each cell, the response variation is defined as the mean of the Gaussian fit to the response variation distribution of the channel associated with the cell. As seen in Figure 4, the observed down-drift mostly affects cells of the inner radius, particularly A13 cells and cells in the E4 region, all of which are cells with higher current. Figure 5 demonstrates the down-drift over time during data-taking in 2016. It is noteworthy that response variation of A13 cells, 


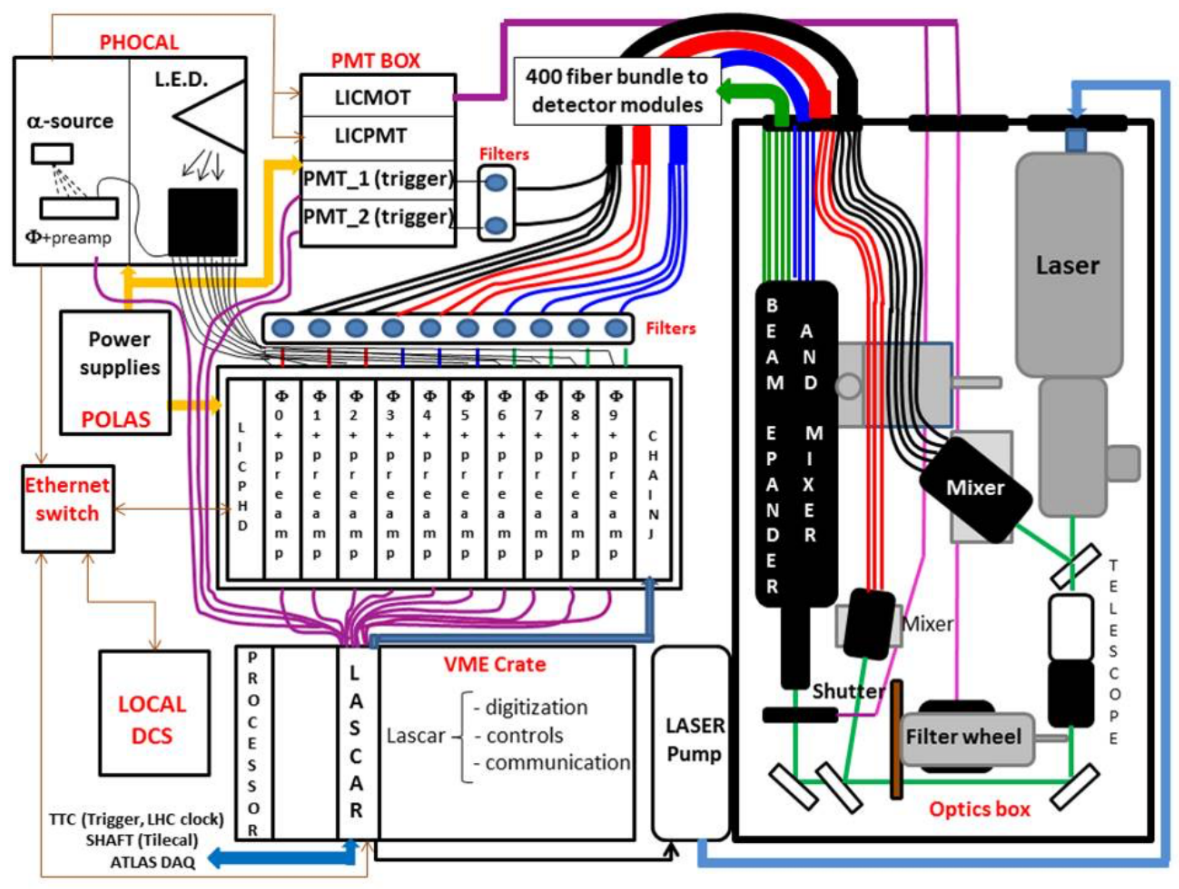

Figure 2: The full Laser II system containing a 432 nm laser

\begin{tabular}{|c|c|c|c|c|c|c|c|c|}
\hline & \multicolumn{4}{|c|}{$\begin{array}{c}\text { Short term stability } \\
\text { (1 month, between two Cs scans) }\end{array}$} & \multicolumn{4}{|c|}{$\begin{array}{c}\text { Long term stability } \\
\text { ( } 4 \text { month observation ) }\end{array}$} \\
\hline & \multicolumn{2}{|c|}{ RMS } & \multicolumn{2}{|c|}{ maximum } & \multicolumn{2}{|c|}{ RMS } & \multicolumn{2}{|c|}{ maximum } \\
\hline & LG & HG & LG & HG & LG & HG & LG & $\mathrm{HG}$ \\
\hline Laser monitors & 0.3 & 0.3 & 0.5 & 0.5 & 0.5 & 0.5 & 1.5 & 1.5 \\
\hline Filter monitors & 0.3 & 1.0 & 1.0 & 5.0 & 0.5 & 1.5 & 2.0 & 6.0 \\
\hline Expander monitors (\%) & 0.3 & 0.5 & 1.0 & 1.5 & 0.5 & 1.0 & 2.0 & 3.0 \\
\hline Overall & 0.3 & 0.5 & 1.0 & 2.0 & 0.5 & 1.0 & 2.5 & 4.0 \\
\hline
\end{tabular}

Figure 3: Short and long term stabilities of the Laser II system

as seen in the spread of Figure 6, is consistent with Hamamatsu's response over operating time.

The down-drift of PMT response indicates an exponential decay, which allows us to calculate decay constants from the available measurement at the 


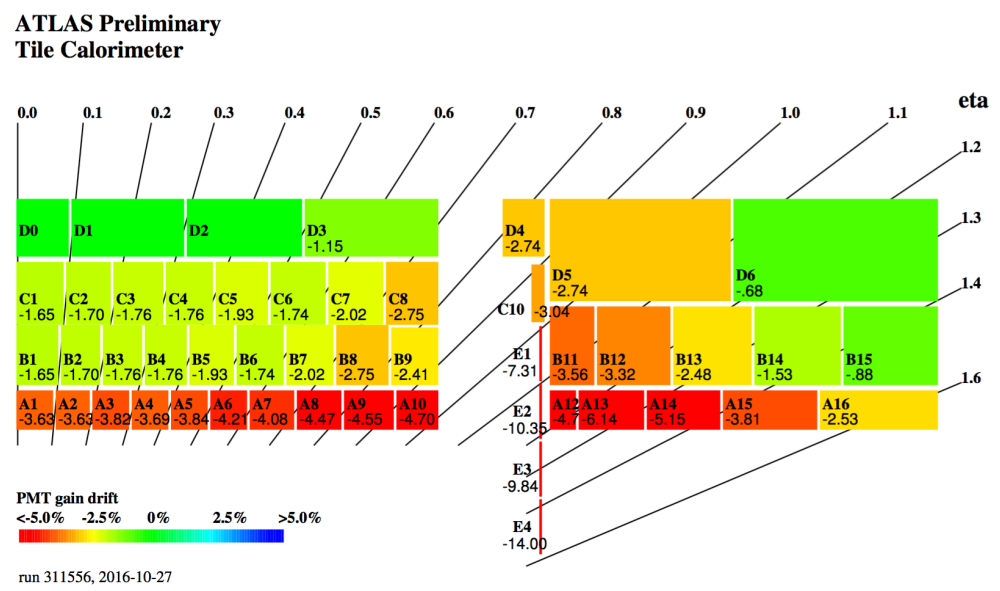

Figure 4: Variation of mean response over 5 months for 10,000 PMTs

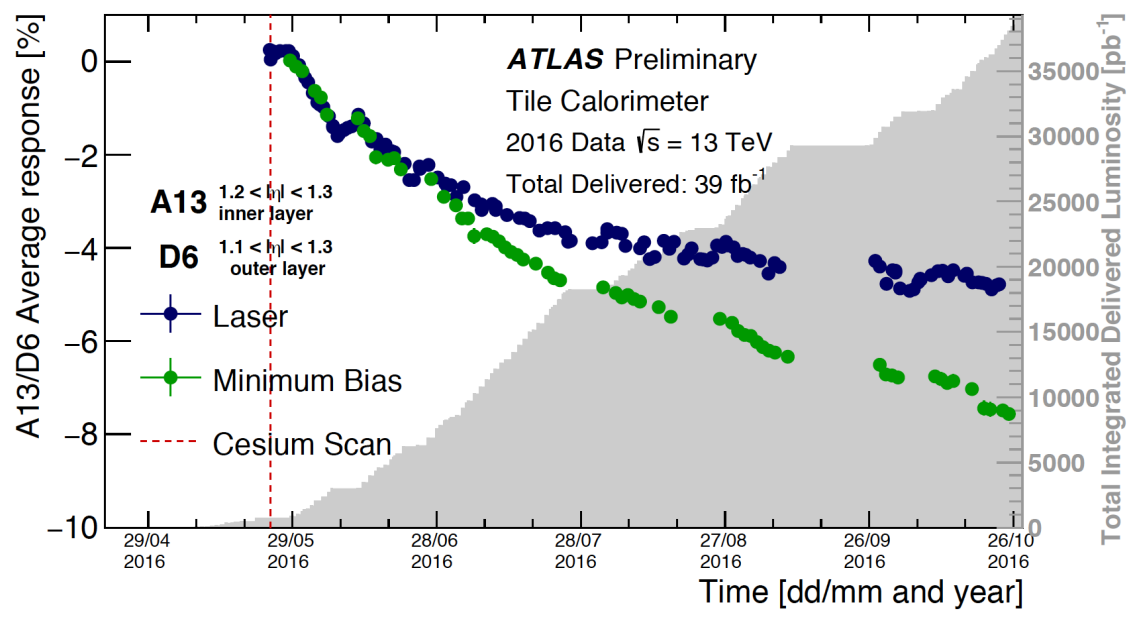

Figure 5: Evolution of down-drift of A13 cells during 2016 data collection

end of Run 1 and after 20 and $35 \mathrm{fb}^{-1}$ in Run 2. These constants make it possible to estimate response loss in the future. As seen in Figure 7, at the end of the HL-LHC era, more exposed PMTs will have lost approximately $50 \%$ of their response. Yet these estimates are not large enough to merit detector-wide PMT replacements. 


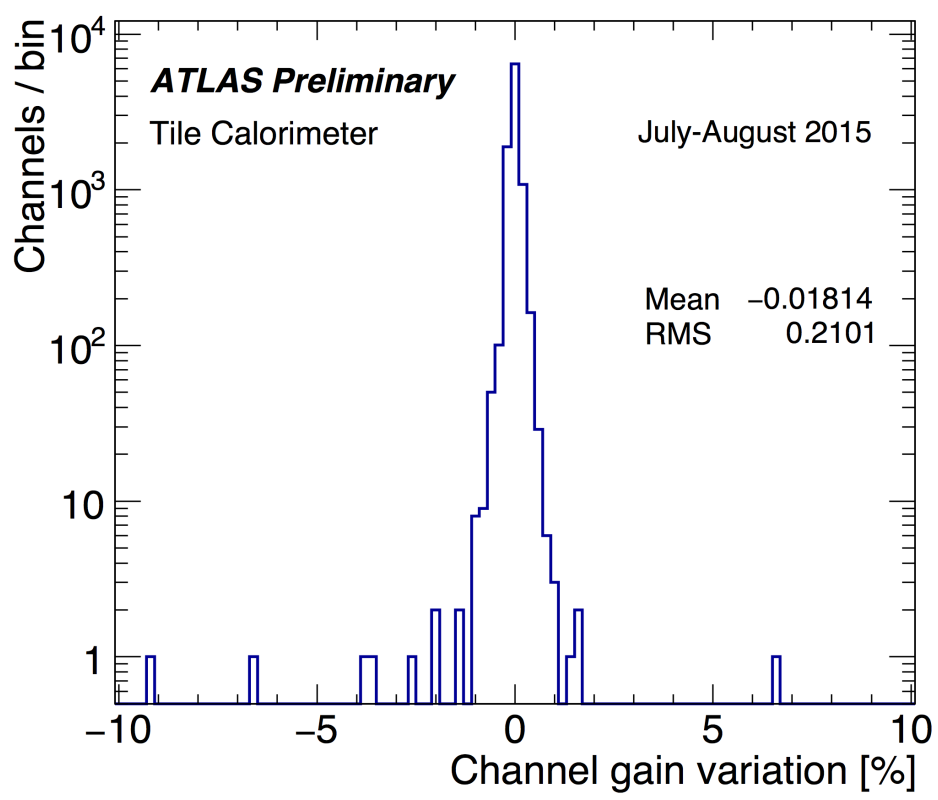

Figure 6: The response variation distribution of A13 cells



Figure 7: Available data indicate that PMT response degrades exponentially, allowing for the estimation of future response loss

\section{Absolute gain measurements}

The response down-drift study of PMTs was repeated for the test bench, as pictured in Figure 8. For the test bench, two methods were applied to 
measure the laser coherence constant $\kappa$ for the absolute gain $G_{i}$ measurement of a $\mathrm{PMT}_{i}[6]$.

$$
\begin{gathered}
G_{i}=\frac{1}{e \cdot f} \cdot \frac{\operatorname{Var}\left(q_{i}\right)}{\left\langle q_{i}\right\rangle}-\frac{1}{e} \kappa\left\langle q_{i}\right\rangle \\
\kappa=\frac{\operatorname{Cov}\left(q_{i}, q_{j}\right)}{\left\langle q_{i}\right\rangle\left\langle q_{j}\right\rangle} \\
\kappa=\frac{\frac{\operatorname{Var}\left(q_{n}\right)}{\left\langle q_{n}\right\rangle}-\frac{\operatorname{Var}\left(q_{m}\right)}{\left\langle q_{m}\right\rangle}}{\left\langle q_{n}\right\rangle-\left\langle q_{m}\right\rangle}
\end{gathered}
$$

$\langle q\rangle$ is the average anode charge, $f$ is the noise excess factor, and $e$ is the electron charge. For the energy scan method (Eq. 3), $n$ and $m$ refer to the same PMT at two different light intensities. For the covariance method (Eq. 2), $q_{j}$ and $q_{i}$ are the anode charges of any PMT pairs receiving similar fractions of the same light pulse at a fixed intensity.

The covariance method, which is based on the correlation between PMT responses, proved to be more accurate than the energy scan method, which was traditionally used in the ATLAS detector. Consequently, the covariance method was applied to the ATLAS detector to calculate $\kappa$ and study gain stability.

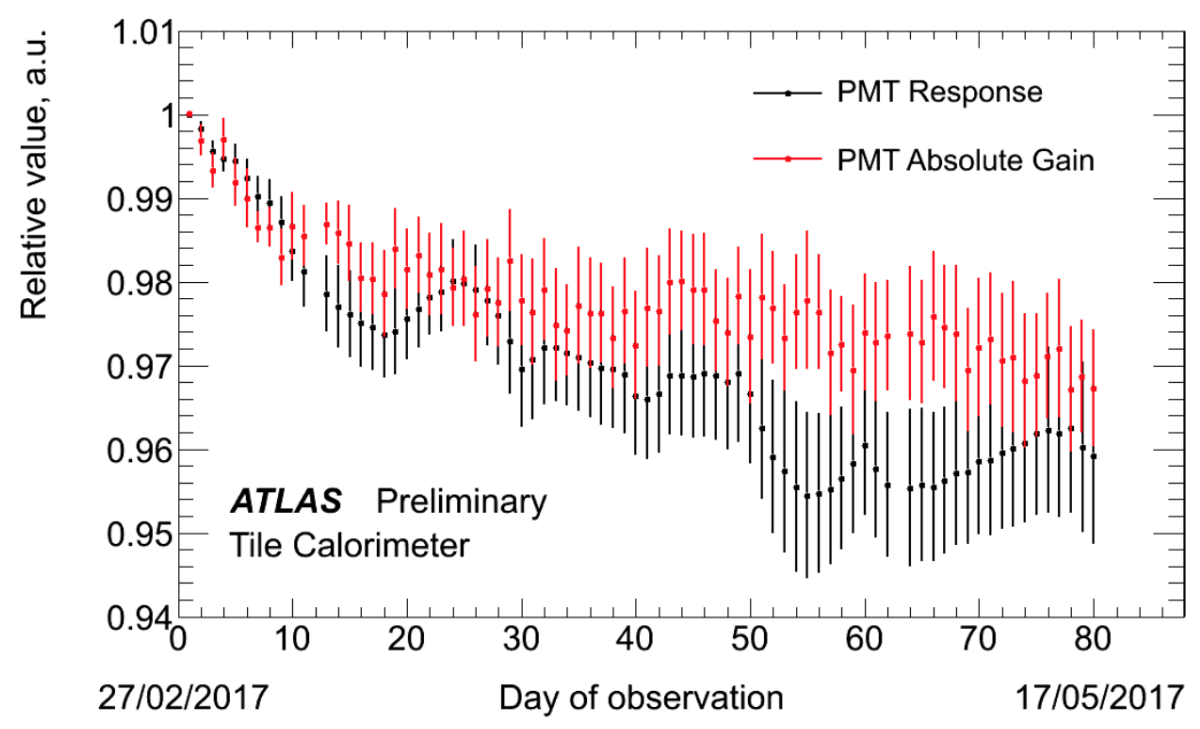

Figure 8: PMT response and PMT absolute gain for the test bench over time 
For the first time, a difference between PMT absolute gain and PMT global response was observed in the test bench measurements with laser excitation. Similar studies will be repeated with Laser calibration data from on-detector PMTs. The difference between absolute gain drift and global response variations can be attributed to additional effects from quantum efficiency and window transparency losses.

\section{Conclusion}

Calculating the approximate exponential decay of the response down-drift in the ATLAS detector allows us to estimate future PMT response loss and validate the decision not to replace PMTs in upcoming upgrades. The test bench demonstrates a measurable correlation between response down-drift and absolute gain down-drift for irradiated PMTs from TileCal. Due to improved sensitivity of the covariance method, we are now also able to more accurately monitor drifts of absolute gain and PMT response in the ATLAS detector.

\section{Acknowledgements}

This work was carried out by the TileCal calibration group. Thank you to everyone who conducted the detector and test bench PMT response studies, particularly the members of the Laser calibration system and the Pisa-INFP labs.

[1] D. Boumediene, on behalf of the ATLAS collaboration Calibration of the Tile Hadronic Calorimeter of ATLAS at LHC. J. Phys.: Conf. Ser. 587 012009, 2015.

http://iopscience.iop.org/article/10.1088/1742-6596/587/1/012009/pdf

[2] P. Martins, F. Manuel The ATLAS Tile Calorimeter DCS for Run 2. IEEE Nuclear Science Symposium and Medical Imaging Conference, Strasbourg, France, 29 Oct - 6 Nov 2016 https://cds. cern. ch/record/2235813

[3] ATLAS Collaboration, Tile Calorimeter Plots. https://twiki.cern.ch/twiki/bin/view/AtlasPublic/ApprovedPlotsTile 
[4] F. Scuri, on behalf of the ATLAS Tile Calorimeter System Performance of the ATLAS Tile LaserII Calibration System. ATL-TILECAL-PROC2015-020, 2015.

https://cds.cern.ch/record/2104005

[5] M. Cornelis van Woerden, on behalf of the ATLAS Tile Calorimeter System Upgrade of the Laser calibration system for the ATLAS hadronic calorimeter TileCal. Frontier Detectors for Frontier Physics: 13th Pisa Meeting on Advanced Detectors, La Biodola, Isola D'elba, Italy, 24 - 30 May 2015, pp.64-66

https://cds. cern. ch/record/2026050

[6] V. Kazanin, S. Leone, and F. Scuri Laboratory tests of the response stability of the ATLAS Tile Calorimeter photomultipliers. ATL-TILECALSLIDE-2017-086, Mar. 2017.

https://cds.cern. ch/record/2254989 